\title{
Emotion Analysis in Gasoline Consumption
}

\section{Dafne Rosso Pelayo ${ }^{1, a}$, Joel Armando Colín Pacheco ${ }^{2, b}$}

\author{
Luis Miralles-Pechuán ${ }^{3, c}$
}

${ }^{1}$ Engineering Department, Universidad Panamericana, Felix cuevas CD MEX, México;

${ }^{2}$ Engineering Department, Universidad Anáhuac del Norte, CD MEX, México;

${ }^{3}$ Engineering Department, Universidad Panamericana, Felix cuevas CD MEX, México ;

adrosso@up.edu.mx , b jacolinp@yahoo.com, ${ }^{\mathrm{c}}$ Imiralles@up.edu.mx

Keywords: Emotion analysis, pattern behavior, gasoline consumption.

\begin{abstract}
Emotions Analysis is a market intelligence technique used to analyze opinions of people about certain company aspects such as product image, product consumption, marketing campaigns, clients' preferences and social or political movements. The relevance of Emotions Analysis research lies in the enormous economic impact that it provides to enterprises. The role played by emotions in decision making has been analyzed in extensive way to ensure its influence in human behavior. In this work, we present an emotion analysis to obtain the principal characteristics set related to the emotions that make consumers prefer a gas station over others in Mexico. We use a statistical approach to analyze the characteristics of gas stations preferred by customers based on their emotions.
\end{abstract}

\section{Introduction}

Emotions are sensations generated by feelings and perceptions, and are accompanied with thinking and actions; they are always accompanied by thoughts [1]. Emotions are the result of brain stimulation and reflect an important impact in clients' decisions [2, 3, 4, 5, 6]. When emotions are positive, the likelihood of finding positive action is higher; otherwise when the emotion is negative the likelihood of finding positive action is lower. Damasio in [7] shows that "basic emotions like happiness, sadness, shame and empathy, are a set of complex chemical responses as neurological both forming a distinctive pattern" [8,9]. There is a lot of research on how emotions affect customer preferences [10, 11]. However, few investigations focus on gasoline consumption. Turrentine, Kurani and Heffer in [4] found that consumers do not budget, manage or track fuel costs. They also discovered that fuel economy decisions are based more by emotions than by critical analysis and that they are more influenced by social awareness than by its monetary value [4]. This social awareness is also related to social responsibility on environmental issues. In [5] it is concluded that consumer behavior regarding gasoline products is affected substantially within environmentally sensitive target groups. Emotional experience with product's brands is also an important element that affects customers' choice. In [6] Hansen, Christensen and Lundsteen state that when a memory is recalled, all of its components get together and the emotional association with the brand comes up too; these emotional responses are the frame of conscious cognitive process. Our research analyzes customers' preferences on gas stations from two perspectives. Firstly we use a statistical analysis in order to explore the distribution of the information and to observe the behavior of the gas station selection process according to clients' perceptions; which are originated from clients' experiences when they consume gasoline. Secondly we perform a statistical analysis to model the behavior of people selecting a particular gas station based in their emotions; which are generated when they consume the gas stations' services.

\section{Conventions}

Customer behavior is represented by an independent object characteristic $x_{i}$ that derives a particular emotion $e_{i}$. The combination of dependent or independent characteristics $x_{\mathrm{i}}$ can lead to other 
particular emotion $e_{i}$, where $e_{i} \in E$ and $\mathrm{E}$ is the set of possible emotions. In this way client's feelings and perceptions over any $x_{i}$ become an input to produce $e i$. Each gas station $G_{i}$ possesses a feature set $X$ of size $m$. These features are independent and each feature $x_{i}$ has been rated for each client. We have defined a set of personal clients' features $\mathrm{Y}$, where each $y_{i} \in Y$ represents a client feature i.e. $y_{i}=$ age, gender, etc. $\}$. Each feature $x_{i} \in X$ represents a feature that clients can get from $G_{i}$ during the service time, i.e. $x_{i}=\{$ clean bathrooms; store; etc. $\}$.

\section{Dataset Description}

The dataset used was obtained by an online survey answered in social networks only by Mexicans that consume gasoline. We represent the vehicles population in Mexico by MV according to the census of the "INEGI", in 2014. Thus we obtained from $M V$ a representative sample of the population $S$ of size $n$, where $S \in M V$, of enough size according to (1) to obtain statistically significant results, we select a constant value for standard deviation $\sigma$, a 95\% confidence level with $z=1.96$, and an error $e$ of $0.056 \%$. The sample size was estimated according statistical principles with the following formula.

$$
n=\frac{Z^{2} \sigma^{2} N}{e^{2}(N-1)+Z^{2} \sigma^{2}}
$$

A set of personal features $Y$ and a vector of ranked features $R$, where $\mathrm{r}$ is a ranked feature, $r \in R$, were obtained for every client $\mathrm{tj}$. Where $r(i, j)$ is the rating given from a client $\mathrm{c}_{j}$ to a gas station feature $x_{i}$ with $x \in X$, where $X$ represents the set of gas station's features evaluated. The ratings $r(i, j)$ varies in an interval $[1,10]$ where " 1 " is the minimum rating and " 10 " is the maximum rating.

\section{Statistical Analysis}

Our statistical analysis reflects the behavior of the independent variable represented by personal characteristics of clients $Y$ over their consumption using ranked gasoline characteristics $x_{i}$. Table 1 represents an example of set $Y$. This set includes clients' personal features, where each $y_{i} \in \mathrm{Y}$ represents a client characteristic.

Table 1. Personal client features

\begin{tabular}{ll}
\hline Client $\mathrm{Ci}$ & \multicolumn{1}{c}{$\mathrm{Yi}$} \\
\hline $\mathrm{y} 1$ & Age \\
$\mathrm{y} 2$ & Gender \\
$\mathrm{yn}$ & Average gasoline consumption \\
\hline
\end{tabular}

In $S$ each gasoline feature $x_{i}$ is related in a supervised way to a particular emotion $e_{i}$ so $S$ can be used to analyze characteristics $x_{i}$, which had been rated by clients, and to discover the most relevant emotions that drive clients to select a specific gas station. The analysis was performed to every $x_{i}$ and every personal feature as well as their combinations. This shows that when one characteristic with a high rating is presented with another one with low rating clients still prefer the gas station based on the higher one as shown in Figure 1. Low rated characteristics correlated with other medium or low rated characteristics are not selected, Figure 2. Figures 3 and 4 show how preference varies according to clients' profile.
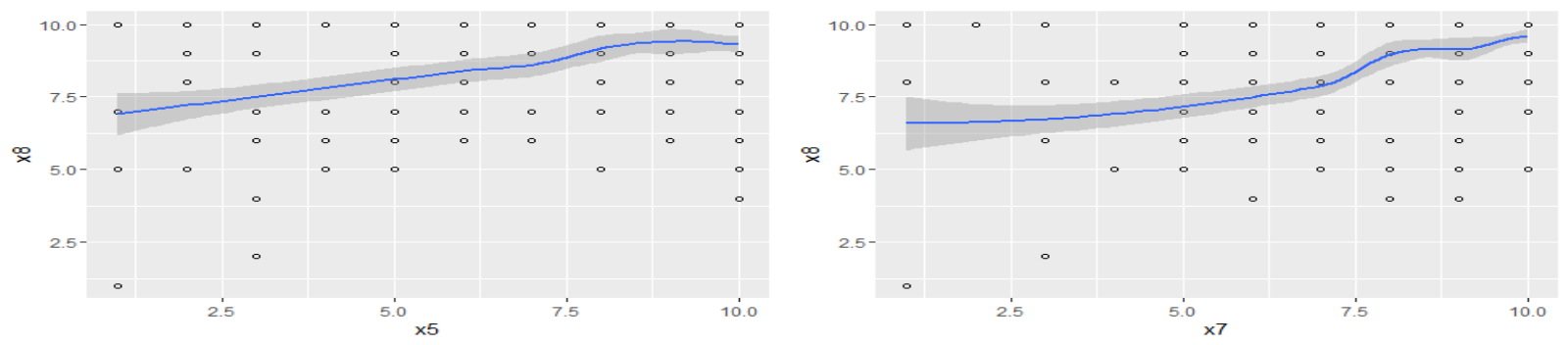

Fig 1. High rated characteristic vs two low rated characteristics 

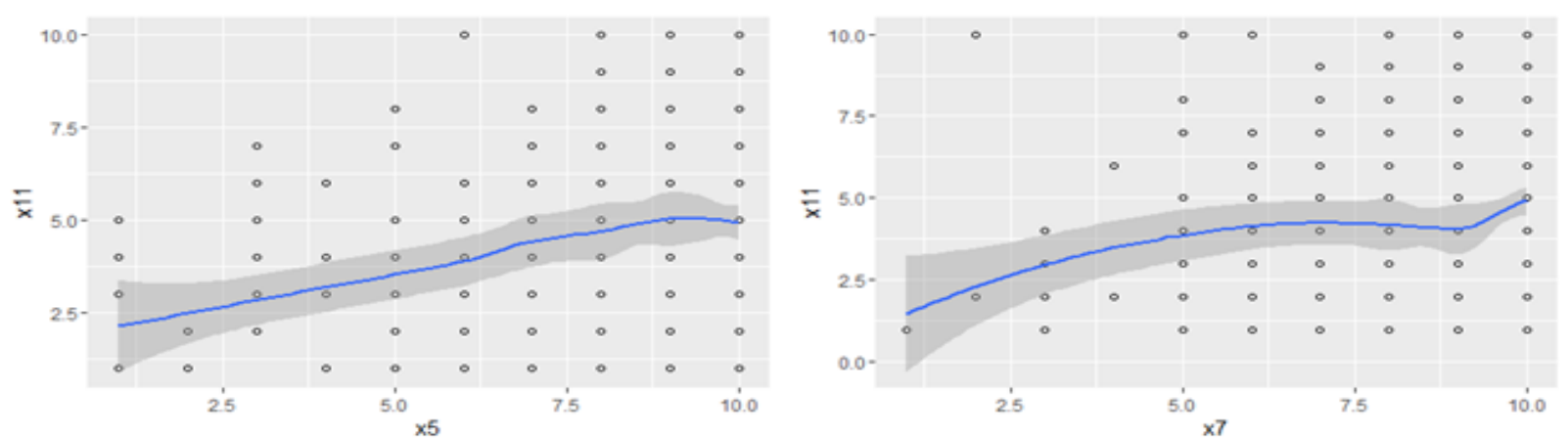

Fig 2. Low rated characteristic vs medium rated characteristic
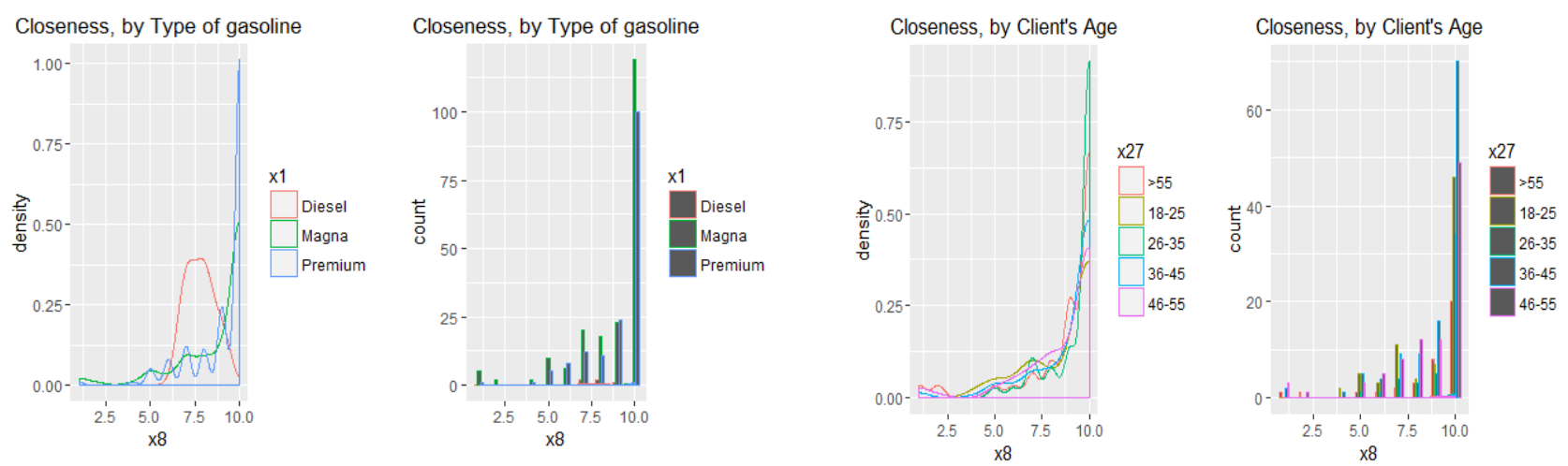

Fig 3. Distinct preferences distribution related to Closeness

This shows that there are some variables that are critical in the selection of the gas station. Also we identify that some characteristics are not equally relevant by segment, and that clients prefer other kind of intrinsic characteristics instead of additional services as shown in Figure 2. In the second part of our analysis, emotion variables were added in association with the characteristics. This will show not only the features that clients prefer when they select a gas station, but also those that trigger one or more emotions which influence the selection of a particular gas station.

\section{Data Transformation}

To perform our emotion analysis a transformation process is needed: for every $x_{i}$ we added an associated emotion $e_{i}$, in such a way that personal characteristics are preserved. The $e_{i}$ was mapped to $x_{i}$ based on studies that shows that emotions are produced by external stimulus and they produce an instantaneous impact over people [8]. To distinct between positive and negative emotions we take into account that people exposed to external stimulus incorporate in their neurological patterns those that are beneficial to their life and discard those that are not [10,12]. We took best ranking characteristics as accepted for people and were mapped with positive emotions. After the transformation process we call our new data set as S'.

The $e_{i}$ was assigned according to the type of characteristic, nevertheless the set of emotions of a single client has a particular combination and proportion of emotions and characteristics that make him to select or not select a specific gas station.

Table 2. Example of client Emotions in S'

\begin{tabular}{lll}
\hline Client $\mathrm{C}_{\mathrm{i}}$ & $\mathrm{x}_{\mathrm{i}}$ & $\mathrm{e}_{\mathrm{i}}$ \\
\hline $\mathrm{e}_{1}$ & Automatic charge & Security \\
$\mathrm{e}_{\mathrm{n}}$ & Rapid service & Happiness \\
\hline
\end{tabular}

Figure 4 shows how emotions are related to gas station features in the selection (rated $>=5.0$ ) or in the rejection (rated $<5.0$ ) of a gas station. 


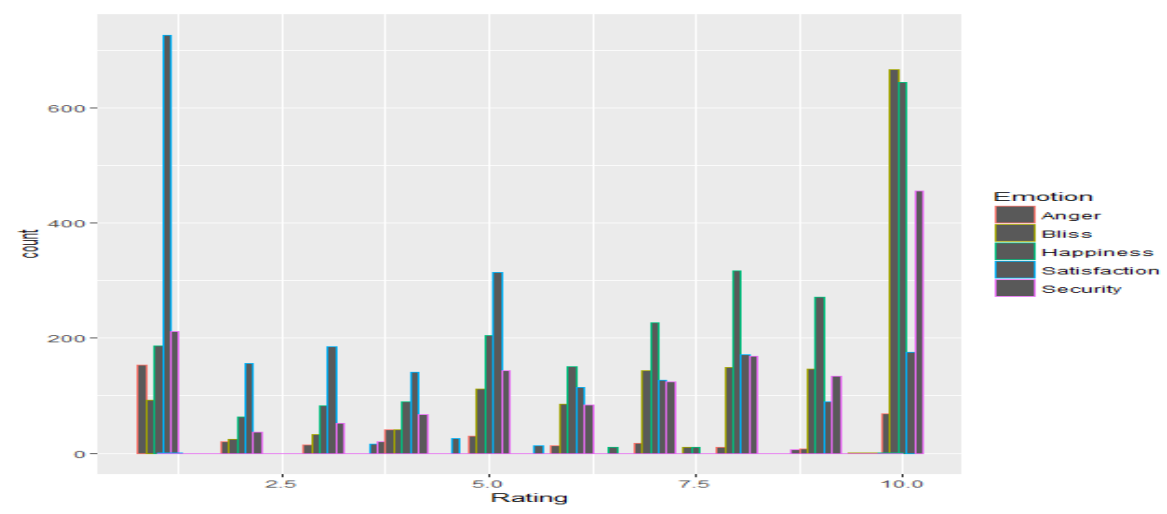

Fig 4. Rating by emotions

\section{Conclusions and Future work}

In this work we show how client decisions are influenced by the emotions produced from the gas station characteristics. We show that a statistical approach can obtain the probability of selecting a gas station over another based on the emotions it produces in customers. A future work of this study would implement Machine Learning approaches to predict client preferences over competency, emphasizing the gas station characteristics in adequate proportions according to the preferences of clients and their emotions.

\section{References}

[1] Ratner, C. A cultural-psychological analysis of emotions. Culture \& Psychology, 6(1), (2000) 5-39.

[2] Frijda, N. H., Manstead, A. S., \& Bem, S. Emotions and beliefs: How feelings influence thoughts. Cambridge University Press. (2000).

[3] Cambria, E., \& Melfi, G. Semantic Outlier Detection for Affective Common-Sense Reasoning and Concept-Level Sentiment Analysis. In FLAIRS Conference (2015, April). (pp. 276-281).

[4] Turrentine, T., Kurani, K. S., \& Heffner, R. R. Fuel economy: what drives consumer choice?. Institute of Transportation Studies. (2008).

[5] Singh, S., Vrontis, D., \& Thrassou, A. Green marketing and consumer behavior: The case of gasoline products. Journal of Transnational Management, 16(2), (2011). 84-106.

[6] Hansen, F., Christensen, S. R., \& Lundsteen, S. Measuring emotions in a marketing context.Innovative Marketing, 2 (2), (2006). 68-75.

[7] Damasio, Antonio R. En busca de Spinoza: neurobiología de la emoción y los sentimientos. Ed. Crítica, S.L., 2005.

[8] Goleman, Daniel. Inteligencia emocional. Editorial Kairós, 2012.

[9] Ahn, Hyungil, and Rosalind W Piccard. Affective-cognitive learning and decision making: A motivational reward framework for affective agents. International Conference on Affective Computing and Intelligent Interaction. Springer Berlin Heidelberg, p. (2005) 866-873.

[10] Ferrer, Alba. Neuromarketing, la tangibilizacion de las emociones. Universidad Abat Oliba CEU, Francia, 2009.

[11] Minsky, M. The emotion machine: Commonsense thinking, artificial intelligence, and the future of the human mind. Simon and Schuster. (2007).

[12] Aurier, Philippe, and Guergana Guintcheva. The Dynamics of Emotions in Movie Consumption: A Spectator-Centred Approach. International Journal of Arts Management 17.2 (2015): 5. 